\title{
Establishment of introduced reptiles increases with the presence and richness of native congeners
}

\author{
Rodrigo B. Ferreira*, Karen H. Beard, Stephen L. Peterson, Sharon A. Poessel, Colin M. Callahan
}

\begin{abstract}
Darwin proposed two contradictory hypotheses to explain the influence of congeners on the outcomes of invasion: the naturalization hypothesis, which predicts a negative relationship between the presence of congeners and invasion success, and the pre-adaptation hypothesis, which predicts a positive relationship between the presence of congeners and invasion success. Studies testing these hypotheses have shown mixed support. We tested these hypotheses using the establishment success of non-native reptiles and congener presence/absence and richness across the globe. Our results demonstrated support for the pre-adaptation hypothesis. We found that globally, both on islands and continents, establishment success was higher in the presence than in the absence of congeners and that establishment success increased with increasing congener richness. At the life form level, establishment success was higher for lizards, marginally higher for snakes, and not different for turtles in the presence of congeners; data were insufficient to test the hypotheses for crocodiles. There was no relationship between establishment success and congener richness for any life form. We suggest that we found support for the pre-adaptation hypothesis because, at the scale of our analysis, native congeners represent environmental conditions appropriate for the species rather than competition for niche space. Our results imply that areas to target for early detection of non-native reptiles are those that host closely related species.
\end{abstract}

Keywords: biological invasions, Darwin's naturalization hypothesis, herpetofauna, introduced species, invasive species, preadaptation hypothesis.

\section{Introduction}

Darwin (1859) implicitly proposed two longstanding and seemingly contradictory hypotheses regarding the ability of non-natives to establish in new environments. In the "naturalization hypothesis", Darwin (1859, p. 114) proposed that, because native and non-native congeners have shared traits and exploit similar ecological niches, it is more difficult for non-natives to adapt to and establish in novel environments when native congeners are present (Rejmanek, 1996). In the "pre-adaptation hypothesis", Darwin $(1859$, p. 86$)$ proposed that invaders are more likely to be successful in areas where native congeners are present because in these areas non-native species are likely to contain traits that pre-adapt them to the new environment (Daehler, 2001; Duncan and Williams, 2002).

Department of Wildland Resources and the Ecology Center, Utah State University, Logan, Utah, 843225230, USA

*Corresponding author; e-mail: rodrigoecologia@yahoo.com.br
To date, plants have been the focus of most studies testing these hypotheses, but these studies have produced mixed results. Some studies supported Darwin's naturalization hypothesis (Mack, 1996; Rejmanek, 1996; Rejmanek, 1998), while others supported the preadaptation hypothesis (Daehler, 2001; Duncan and Williams, 2002), and still others supported neither hypothesis (Lambdon and Hulme, 2006). We are aware of only two studies that have tested these hypotheses with animals at a global scale. Ricciardi and Mottiar (2006) did not find support for either hypothesis for fish invasions, and Tingley, Phillips and Shine (2011) tested the hypotheses for amphibians using both congener presence and richness, and found support for the pre-adaptation hypothesis. Van Wilgen and Richardson (2011) conducted the first test of these hypotheses for reptiles in Florida and California, USA, and showed support for the naturalization hypothesis. To our knowledge, there is no study analyzing these hypotheses at a global scale for reptiles. 
Due to the importance of reptiles as pets and as invasive species, testing Darwin's hypotheses is especially important for this taxon (Kraus, 2009; van Wilgen and Richardson, 2011). Different life-history traits in reptiles (some aquatic, some terrestrial, and others omnipresent) may make it so reptiles can invade a wide spectrum of habitats. Furthermore, some reptiles present health and economic risks to humans, and may require additional management. Understanding the relationship between native congeners and the establishment of nonnative reptiles can contribute to the development of proactive management strategies, particularly the adoption of strategies that prevent introduction and establishment (Kolar and Lodge, 2001).

Here we test Darwin's naturalization hypothesis and pre-adaptation hypothesis for reptiles by examining the influence of congener presence/absence and richness on establishment success and failure of non-native reptile species across global locations. To assess whether there was a difference between islands and continents, we examined them separately. Finally, we investigated these hypotheses for each reptile life form separately and for each continent separately.

\section{Materials and methods}

To test these hypotheses, data on successful and unsuccessful establishment of reptile species across the globe were taken from Kraus (2009). This database has been used by other studies (e.g., Bomford et al., 2009; Tingley, Phillips and Shine, 2011; van Wilgen and Richardson, 2011, 2012) and contains the large majority of the world's published accounts of introductions involving reptiles. Only introductions that took place outside of a species' native geographic range are included in the database.

Within the database, many records consist of multiple introductions of the same species to the same locality, but we considered establishment successful if at least one of these introductions was successful (as in Bomford et al., 2009). Taxa lacking identification at the species-level and unknown outcomes of the introduction (established or failed) were removed from our analysis. We only included species where establishment could be labeled as either successful (yes) or failed (no).
Our analysis included 1312 introductions of 405 species that were introduced at least once outside their native geographic ranges. Introductions were considered for a total of 251 localities globally, mostly at the country-level, except for the United States and Canada, which were analyzed at the state- or province-level. Data for countries that exist on the same island (i.e., Haiti and the Dominican Republic) were combined for the analysis. We separated introductions by geographical region: Africa, Asia, Caribbean, Europe, Oceania, Mesoamerica, North America, and South America. Mesoamerica included countries of Central America plus Mexico. In addition to the data provided by Kraus (2009), we collected presence/absence and richness of native reptile congeners for each location using the Reptile Database (http://reptile-database.reptarium.cz; Uetz et al., 2007).

All analyses were conducted in SAS/STAT v. 9.3 for Windows. The two invasion hypotheses were tested for all reptiles globally, for islands and continents, and for each region (Africa, Asia, Caribbean, Europe, Oceania, North America, and South America) separately using Fisher's exact test (as in Ricciardi and Mottiar, 2006). Mesoamerica did not have any unsuccessful introductions, so we were unable to conduct a Fisher's exact test. A significant lefttailed test supported the naturalization hypothesis that successful establishment was more likely in the absence of congener species. A significant right-tailed test supported the pre-adaptation hypothesis that successful establishment of non-native reptiles was more likely in the presence of congener species. The two invasion hypotheses were then tested for each life form separately, except for crocodiles, which lacked adequate data to test the hypotheses. We adopted the term "life form" instead of taxonomic group to designate snakes, lizards, turtles, or crocodiles because snakes and lizards are a monophyletic group (as in van Wilgen and Richardson, 2012). To test for species over-representation in our database, we removed the two most represented genera and re-ran the analyses testing both hypotheses. For lizards, we removed Anolis and Hemidactylus (37\% of the database for lizards). For snakes, we removed Elaphe and Ramphotyphlops (30\% of the database for snakes). For turtles, we removed Mauremys and Trachemys (33\% of the database for turtles). The hypotheses were tested using the PROC FREQ procedure.

Logistic regression was used to assess the relationship between native congener richness and probability of invasion success for all reptiles combined, on continents and islands separately, for each region separately, and for each life form separately. These tests were conducted using the PROC LOGISTIC procedure.

\section{Results}

Of 1312 reported introductions of reptiles worldwide, $708(54 \%)$ resulted in the establishment of the species while $604(46 \%)$ failed, and 306 introductions $(23 \%)$ occurred in the presence of congeners and $1006(77 \%)$ in the absence of congeners. Non-native reptiles showed 
Table 1. Successful and unsuccessful establishment (percent) of non-native reptiles. $P$-values are for tests of Darwin's naturalization hypothesis (left-tailed), pre-adaptation hypothesis (right-tailed), and congener richness.

\begin{tabular}{|c|c|c|c|c|c|c|c|}
\hline \multirow[t]{2}{*}{ Scale } & \multicolumn{2}{|c|}{ Presence of congener } & \multicolumn{2}{|c|}{ Absence of congener } & \multicolumn{2}{|c|}{ Hypothesis } & \multirow[t]{2}{*}{ Richness } \\
\hline & Successful & Failed & Successful & Failed & Left & Right & \\
\hline Global & $201(66 \%)$ & $105(34 \%)$ & $507(51 \%)$ & $499(49 \%)$ & 1.000 & $<0.001$ & $<0.001$ \\
\hline Island & $96(91 \%)$ & $9(9 \%)$ & $279(57 \%)$ & $207(43 \%)$ & 1.000 & $<0.001$ & $<0.001$ \\
\hline Continent & $105(53 \%)$ & $92(47 \%)$ & $228(44 \%)$ & $296(56 \%)$ & 0.992 & 0.019 & 0.011 \\
\hline
\end{tabular}

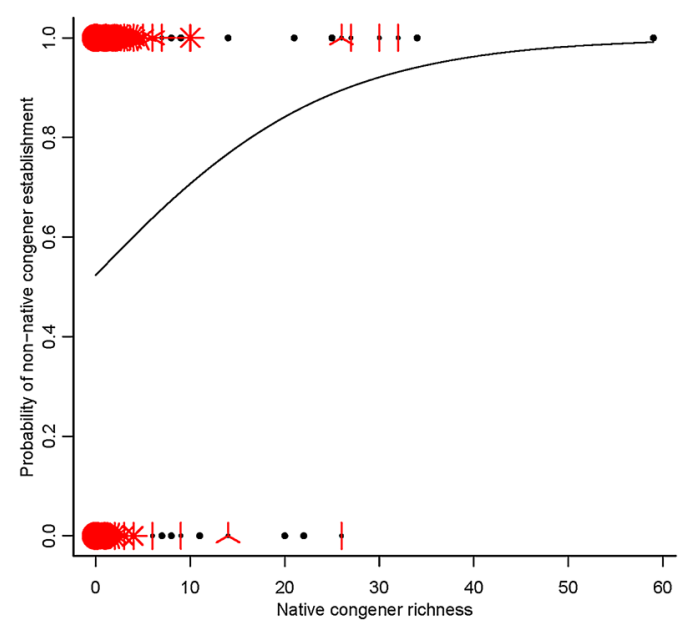

Figure 1. Probability of successful establishment of nonnative reptiles into novel environments with richness of native congener species at a global scale. Multiple points are plotted as "sunflowers" with multiple leaves ("petals") such that overplotting is visualized. Dashed lines show 95\% confidence intervals. This figure is published in colour in the online version.

a higher rate of establishment in localities containing at least one congener species than in localities without congener species (table 1). Furthermore, the probability of successful establishment increased with increasing congener richness (table 1; fig. 1). This result was also robust to the removal of an outlier, 59 congeneric species in Mexico for the introduced Anolis sagrei $(P=0.001)$.

On islands, there were 591 introductions, whereas on continents, there were 721 introductions. The presence of congeners was significantly related to establishment success on both islands and continents (table 1). Additionally, richness of congeners was a significant factor for successful establishment on islands and on continents (table 1).
The percentages of non-native reptiles introduced into each geographic region were: Africa (8\%), Asia (8\%), Caribbean (9\%), Europe (19\%), Mesoamerica (3\%), North America $(34 \%)$, Oceania (14\%), and South America $(5 \%)$. Establishment of non-native reptiles in the Caribbean $(P=0.001)$, Europe $(P=$ $0.001)$, and Oceania $(P=0.048)$ was more successful in the presence of congeners. The other regions (Africa, Asia, North America, and South America) did not show support for either hypothesis. Richness was a significant factor for successful establishment only in Europe $(P<0.001)$.

Lizards represented $48 \%$ (627) of all introductions, while snakes and turtles represented $25 \%$ each (332 and 331 introductions, respectively). Crocodiles with only 22 introductions were the least introduced reptile group representing only $2 \%$ of all introductions (table 2 ). Establishment success was higher for lizards, marginally higher for snakes, and not different for turtles in the presence of congeners (table 2). When the two most represented genera of each life form were removed from our analysis, snakes were the only group that showed a difference compared to the previous results. In this new analysis, in which we attempted to account for over-representation, establishment success of snakes was even higher in the presence of congeners $(P=0.008)$. Richness of congeners was not an important factor in predicting successful establishment for lizards, snakes, or turtles at the global scale (table 2). 
Table 2. Successful and unsuccessful establishment (percent) of non-native reptiles by life form. $P$-values are for tests of Darwin's naturalization hypothesis (left-tailed), pre-adaptation hypothesis (right-tailed), and congener richness. -, hypothesis not tested.

\begin{tabular}{|c|c|c|c|c|c|c|c|}
\hline \multirow[t]{2}{*}{ Order } & \multicolumn{2}{|c|}{ Presence of congener } & \multicolumn{2}{|c|}{ Absence of congener } & \multicolumn{2}{|c|}{ Hypothesis } & \multirow[t]{2}{*}{ Richness } \\
\hline & Successful & Failed & Successful & Failed & Left & Right & \\
\hline Crocodiles & 0 & 0 & $3(14 \%)$ & $19(86 \%)$ & - & - & - \\
\hline Lizards & $145(82 \%)$ & $31(18 \%)$ & $298(66 \%)$ & $153(34 \%)$ & 1.000 & $<0.001$ & 0.137 \\
\hline Snakes & $30(44 \%)$ & $38(56 \%)$ & $88(33 \%)$ & $176(67 \%)$ & 0.963 & 0.066 & 0.123 \\
\hline Turtles & $26(42 \%)$ & $36(58 \%)$ & $118(44 \%)$ & $151(56 \%)$ & 0.448 & 0.661 & 0.162 \\
\hline
\end{tabular}

\section{Discussion}

Globally, establishment success of non-native reptiles was higher in the presence of congeners and increased with increasing congener richness in novel environments. Reptiles showed greater establishment success in the presence of congeners both on island and continent localities, thus following the global pattern. These results supported the pre-adaptation hypothesis rather than Darwin's naturalization hypothesis.

Of the two previous global assessments of congener effects on animal taxa, one found support for the pre-adaptation hypothesis (Tingley, Phillips and Shine, 2011), and the other found no support for either hypothesis (Ricciardi and Mottiar, 2006). Our results were similar to those of Tingley, Phillips and Shine's (2011) that analyzed amphibian invasions, although these authors incorporated more variables. Combining our results with Tingley, Phillips and Shine's (2011) results allows us to conclude that for herpetofauna, in general, establishment success is higher in the presence of congeners and increases with increasing congener richness at the global scale. Our results differed from those of van Wilgen and Richardson (2011, 2012), who found, with weak support, that successfully established reptiles were more distantly related to natives than were failed species in California and Florida.

Differences in the influence of congeners on establishment success among life forms were expected due to the diversity of life-history and behavioral characteristics across reptile life forms. For lizards and snakes (after removing the two most represented genera), native con- geners were an important predictor of establishment success, and because lizards made up the majority of reptile introductions, they likely drove the pattern at the global scale. Snakes showed a higher proportion of failed establishment in the presence and in the absence of native congeners than lizards. In general, snakes are considered the most harmful reptile taxa for humans, so governmental efforts may target snakes, which may influence their rates of establishment (Bomford et al., 2009).

Lower sample sizes or more specific habitat requirements of turtles may explain why they did not support either hypothesis. Turtles often require aquatic habitat, and therefore their establishment success may be greatly reduced in localities with scarce fresh water sources. A study conducted at a finer spatial scale may be appropriate to reveal the relevance of congeners for turtles.

Our analysis does not indicate why introduced reptiles were more likely to be successful where there were native congeners; however, as Darwin first proposed, this result likely occurs because congeneric species should have similar traits, and thus congeners should indicate "pre-adaptation" for the introduced species to the local environmental conditions. For example, invaders may be more successful where congeners are present because congeners indicate that physiological tolerances are compatible with local conditions (MacIsaac, Grigorovich and Ricciardi, 2001). In support of this, van Wilgen and Richardson (2011) noted that for native and non-native reptiles in Florida and California phylogenetic similarity repre- 
sents ecological similarity, although that particular study used molecular phylogenies and not congener status, which is a crude measure of phylogenetic relatedness. Alternatively, fundamental niches can be very different between two species even in the same genus (van Wilgen and Richardson, 2011, 2012).

Because of the scale of our analysis, our findings likely do not indicate outcomes associated with direct species interaction (e.g., competition or predation) but rather indicate habitat and abiotic suitability of the novel environment (van Wilgen and Richardson, 2011, 2012). Thuiller et al. (2010) similarly concluded that direct species interactions likely do not drive patterns at global scales, and that these are more reflective of environment filtering, regional heterogeneity, and species dispersal abilities (also see Duncan and Williams, 2002; Proches et al., 2008; Tingley, Phillips and Shine, 2011).

Future investigations of Darwin's naturalization hypothesis should take into account both the characteristics of invaders (e.g. functional traits related to resource acquisition) and direct biotic interactions (e.g. predation and competition), if possible, at local scales relevant to community interactions. This analysis would determine more precisely how introduced species interact with close indigenous relatives, and under what circumstances these interactions have the most influence. In addition, we acknowledge that there are likely to be factors, such as propagule pressure and climate matching, that may override the importance of phylogenetic relatedness in determining invasion success (Bomford et al., 2009; Van Wilgen and Richardson, 2012). While we did not set out to test these additional hypotheses, they are certainly important to consider in future studies.

There are limitations to a global dataset such as the one used in this study. First, data across life forms are not equally represented. Furthermore, some regions (North America, Europe) are over-represented in the database, as a result of unequal documentation and introductions. Over-representation may influence results in that the results may better describe particular taxa and regions than all taxa equally at the global scale (Sol et al., 2008). To address this issue, we removed dominant genera (around 30\% of the data) for each life form, and conducted analyses separately by region. While some analyses were no longer significant with these modifications to the database, all significant results were in the same direction of the overall analyses suggesting that with the best available database at this scale the general findings were robust. However, we do have to be careful with interpretation of global analyses such as this, and these hypotheses should be revisited as additional information is collected.

Support for Darwin's pre-adaptation hypothesis has interesting management implications in terms of location vulnerability and riskassessment. Because, in general, imported reptile species, and specifically lizards, are more likely to establish in environments that harbor taxonomic relatives, this implies that some communities are more prone to reptile invasion than others. This finding should help wildlife managers to target high-risk areas, and assist in the development of effective management strategies for areas that are most likely to be invaded (McNeely et al., 2001).

Acknowledgements. We thank Susan Durham for statistical advice. We thank Fred Kraus who created the database, and Erika Stevenson who contributed to data collection.

\section{References}

Bomford, M., Kraus, F., Barry, S.C., Lawrence, E. (2009): Predicting establishment success for alien reptiles and amphibians: a role for climate matching. Biol. Invasions 11: $713-724$

Daehler, C. (2001): Darwin's naturalization hypothesis revisited. Am. Nat. 158: 324-330.

Darwin, C. (1859): On the Origin of Species. J. Murray, London.

Duncan, R., Williams, P. (2002): Darwin's naturalization hypothesis challenged. Nature 417: 608-609.

Kolar, C., Lodge, D. (2001): Progress in invasion biology: predicting invaders. Trends Ecol. Evol. 16: 199-204. 
Kraus, F. (2009): Alien Reptiles and Amphibians: A Scientific Compendium and Analysis Series. Springer, Dordrecht.

Lambdon, P., Hulme, P. (2006): How strongly do interactions with closely-related native species influence plant invasions? Darwin's naturalization hypothesis assessed on Mediterranean islands. J. Biogeogr. 33: 1116-1125.

MacIsaac, H., Grigorovich, I., Ricciardi, A. (2001): Reassessment of species invasion concepts: the Great Lakes basin as a model. Biol. Invasions 3: 405-416.

Mack, R. (1996): Biotic barriers to plant naturalization. In: Proceedings of the Ninth International Symposium on Biological Control of Weeds, p. 19-26. Moran, V., Hoffman, J., Eds, University of Cape Town, Rondebosch, Stellenbosch, South Africa.

McNeely, J., Mooney, H., Neville, L., Schei, P., Waage, J. (2001): Global Strategy on Invasive Alien Species. IUCN, Gland.

Proches, S., Wilson, J., Richardon, D., Rejmanek, M. (2008): Searching for phylogenetic pattern in biological invasions. Global Ecol. Biogeogr. 17: 5-10.

Rejmanek, M. (1996): A theory of seed plant invasiveness: the first sketch. Biol. Conserv. 78: 171-180.

Rejmanek, M. (1998): Invasive plant species and invasible ecosystems. In: Invasive Species and Biodiversity Management, p. 79-102. Sandlund, O., Schei, P., Vilken, A., Eds, Kluwer, Dordrecht.

Ricciardi, A., Mottiar, M. (2006): Does Darwin's naturalization hypothesis explain fish invasion? Biol. Invasions 8: 1403-1407.
Sol, D., Vila, M., Kuhn, I. (2008): The comparative analysis of historical alien introductions. Biol. Invasions 10: 1119-1129.

Thuiller, W., Gallien, L., Boulangeat, I., Bello, F., Munkemuller, T., Roquet, C., Lavergne, S. (2010): Resolving Darwin's naturalization conundrum: a quest for evidence. Divers. Distrib. 16: 461-475.

Tingley, R., Phillips, B.L., Shine, R. (2011): Establishment success of introduced amphibians increases in the presence of congeneric species. Am. Nat. 177: 382-388.

Uetz, P., Goll, J., Hallermann, J. (2007): Die TIGRReptiliendatenbank. Elaphe 15: 22-25.

van Wilgen, N., Richardson, D. (2011): Is phylogenetic relatedness to native species important for the establishment of reptiles introduced to California and Florida? Divers. Distrib. 17: 172-181.

van Wilgen, N., Richardson, D. (2012): The roles of climate, phylogenetic relatedness, introduction effort, and reproductive traits in the establishment of non-native reptiles and amphibians. Conserv. Biol. 26: 1-11.

Submitted: March 13, 2012. Final revision received: August 3, 2012. Accepted: August 4, 2012.

Associated Editor: Sylvain Ursenbacher. 